Article

\title{
Sequence length of HIV-1 subtype B increases over time: anal- ysis of a cohort of patients with hemophilia over 30 years
}

\author{
Young-Keol CHO1*, Jung-Eun KIM1, Brian T. FOLEY2
}

\author{
1. Department of Microbiology, Asan Medical Center, University of Ulsan College of Medicine, Seoul 05505, \\ South Korea; kimje2000@nate.com \\ 2. HIV Databases, Theoretical Biology and Biophysics Group, Los Alamos National Laboratory, NM, USA; \\ btf@lanl.gov \\ *Correspondence: ykcho2@amc.seoul.kr; Tel: 82-2-3010-4283; Fax: 82-2-3010-4259 \\ Young-Keol Cho ORCID 0000-0003-0424-8911 \\ Jung-Eun Kim E-mail: kimje2000@nate.com \\ Brian T. Foley E-mail: $\underline{\text { btf@lanl.gov }}$
}

\begin{abstract}
We aimed to investigate whether the sequence length of HIV-1 increases over time. A longitudinal analysis of full-length coding region sequences (FLs) during an HIV-1 outbreak among patients with hemophilia and local controls infected with the Korean subclade B of HIV-1 (KSB) was performed. Genes were amplified by overlapping RT-PCR or nested PCR and subjected to direct sequencing. Overall, 141 FLs were sequentially determined over 30 years in 62 KSB-infected patients. Phylogenetic analysis indicated that within KSB, two FLs from plasma donors $\mathrm{O}$ and $\mathrm{P}$ comprised two clusters together with 8 and 12 patients with hemophilia, respectively. Signature pattern analysis for the KSB of HIV-1 revealed 91 signature nucleotide residues (1.05\%). In total, 48 and 43 signature nucleotides originated from clusters $\mathrm{O}$ and $\mathrm{P}$, respectively. Only six positions contained $100 \%$ specific nucleotide(s) in clusters O and P. Additionally, in-depth FL analysis over 30 years indicates that the KSB FL significantly increased over time before combined antiretroviral therapy (cART) and decreased with cART. The increase occurred due to a significant increase in env and nef genes, originating in the variable regions of both genes. The increase in the sequence length of HIV-1 over time suggests that it has an evolutionary direction.
\end{abstract}

Keywords: full-length coding region sequence; HIV-1; Korean subclade B; sequence length; hemophilia; evolution

\section{Introduction}

We previously conducted a nationwide genetic analysis of HIV-1 with sera from individuals in the early stages of HIV-1 infection (before 1994) to identify the cause of an HIV-1 outbreak among patients with hemophilia in Korea in 1990-1994. These molecular epidemiological studies revealed that viruses from two cash-paid plasma donors were incompletely inactivated in the process of manufacturing clotting factor IX and were identified as the agents of infection among 20 HIV-1-infected patients with hemophilia [1-6]. The viruses in 8 and 12 patients with hemophilia infected with the Korean subclade of HIV-1 subtype B (KSB) originated from plasma donors $\mathrm{O}$ and $\mathrm{P}$, respectively. In these studies [1-7], we conducted an in-depth analysis by genes, but a few genes are yet to be studied. The sequence length significantly affects the extent of clustering in a phylogenetic tree [8-9]. KSB is a distinct, monophyletic clade within HIV-1 subtype B and is presumed to have originated from strains in the USA through a founder effect $[8,10-13]$. The most recent common ancestor is estimated to have been active around 1984 [14]; however, the earliest case was diagnosed in 1988 [2-6]. 
In this study, we identified signature pattern residues in the full-length coding region sequence of KSB (FLs). We also performed a phylogenetic analysis at the FL level in 64 patients, including 20 patients with hemophilia B (HPs) [1-4]. We confirmed a previously postulated epidemiological link between the viruses that infected $20 \mathrm{HPs}$ and 2 plasma donors and the viruses that infected local control patients [2-6]. Additionally, their longitudinal sequence analyses conducted over approximately 30 years indicate that the sequence length in FL significantly increases over time before the administration of combined antiretroviral therapy (cART). It has been reported that the progression in subtype D-infection is four-fold faster than subtype B [15]. The sequence length was nearly the shortest among group $\mathrm{M}$.

To date, our study is one of the most comprehensive and longest longitudinal studies conducted for the examination of HIV-1 subtype B evolution in vivo originated from a single source of HIV-1 [1-6], and its results provide novel insights into the pathogenesis of HIV-1 infection over time.

\section{Materials and Methods}

\subsection{Ethical Statement}

The institutional review board of the Asan Medical Center approved this study's conduct (Code 2012-0390, 4 June 2012). All subjects provided their informed consent for inclusion before participation in the study. The study was conducted in accordance with the Declaration of Helsinki.

\subsection{Patients and samples}

Four HIV-1 infected plasma donors were diagnosed during primary infection in 1990-1992. Their plasma was used to manufacture the domestic clotting factor IX. Viruses from donors $\mathrm{O}$ and $\mathrm{P}$ were incompletely inactivated. The details have been described previously [1-6, 16, 17]. Briefly, 20 patients with hemophilia (HPs 1-20) were diagnosed with HIV-1 infection between 1990 and 1994. HP 21 was infected with HIV-1 via imported factor 9 prior to 1987 and was diagnosed in 1987. In this study, FLs were sequenced from 62 KSB-infected patients, including 3 plasma donors $(\mathrm{O}, \mathrm{P}$, and $\mathrm{R})$ and 20 patients with hemophilia (designated HP 1-20), 1 CRF02-AG, 1 subtype $D$, and 1 subtype B (Table S1). In this study, the FL sequence denotes the length from the start codon of Gag to Nef with the terminal stop codon.

\subsection{RNA/DNA preparation and FL gene amplification}

Blood samples were collected from $20 \mathrm{HPs}$ in 6-month intervals for CD4+ T cell measurements. Sera were used before the year 2000, and peripheral blood mononuclear cells (PBMC) were used after 2000 for PCR amplification.

Total RNA was extracted from $300 \mu \mathrm{L}$ of serum samples using a QIAamp UltraSens Viral RNA kit (Qiagen, Hilden, Germany), and aliquots of $2 \mu \mathrm{L}$ of RNA were reverse transcribed by mixing with $1 \mu \mathrm{L}$ of oligo(dT), $1 \mu \mathrm{L}$ of dNTPs, and $6 \mu \mathrm{L}$ of DEPC treated water, followed by incubating the mixture at $65^{\circ} \mathrm{C}$ for $5 \mathrm{~min}$ and then on ice for $1 \mathrm{~min}$ [2-6]. To each sample $4 \mu \mathrm{L}$ of $5 \mathrm{x}$ buffer, $2 \mu \mathrm{L}$ of $0.1 \mathrm{M}$ DTT, $3 \mu \mathrm{L}$ of DEPC-treated water (Ambion Inc., Foster City, CA), and $1 \mu \mathrm{L}$ of Superscript III reverse transcriptase (Invitrogen, Carlsbad, CA, USA) were added. The samples were incubated for $50 \mathrm{~min}$ at $50{ }^{\circ} \mathrm{C}$. The reaction was terminated by incubation for $5 \mathrm{~min}$ at $85^{\circ} \mathrm{C}$ and then on ice for $1 \mathrm{~min}$.

DNA was extracted from $400 \mu \mathrm{L}$ of PBMC samples using a QIAamp DNA Mini kit (Qiagen, Hilden, Germany), and aliquots of $5 \mu \mathrm{L}$ of DNA were used for the nested PCR.

The vif, vpr, tat/rev, and vpu regions $(1.2-\mathrm{kb})$ were amplified via nested PCR with TaKaRa r-taq (Takara Bio Inc., Shiga, Japan). The first and second PCR reactions were performed in $20 \mu \mathrm{L}$ and $50 \mu \mathrm{L}$ reaction mixtures, respectively. The outer primer pairs were 545 and KMK2, whereas the inner primer pairs were 548F and LA106, respectively (Table 1). After initial denaturation at $95^{\circ} \mathrm{C}$ for $10 \mathrm{~min}, 35$ cycles were run at $95{ }^{\circ} \mathrm{C}$ for $30 \mathrm{~s}$, 
at $52{ }^{\circ} \mathrm{C}$ for $30 \mathrm{~s}$, and at $72{ }^{\circ} \mathrm{C}$ for $2 \mathrm{~min} 30 \mathrm{~s}$, followed by a final extension step at $72{ }^{\circ} \mathrm{C}$ for $10 \mathrm{~min}$. The second PCR was performed with $1 \mu \mathrm{L}$ of the first PCR product; the cycling conditions were set as follows: $95{ }^{\circ} \mathrm{C}$ for $30 \mathrm{~s}, 57{ }^{\circ} \mathrm{C}$ for $30 \mathrm{~s}$, and $72{ }^{\circ} \mathrm{C}$ for $1 \mathrm{~min} 30 \mathrm{~s}$, as well as a final extension at $72{ }^{\circ} \mathrm{C}$ for $10 \mathrm{~min}$. The procedure for the amplification of the remaining genes is described elsewhere [2, 4-6]. A maximum of five PCR reactions, including that using a negative control, were performed per sample at a given time. To avoid selection bias, all positive PCR amplicons were sequenced and used for the FL. Amplification was performed via five overlapping PCR (Table 1) [16, 17], followed by direct sequencing using the Applied Biosystems 3730XL DNA Analyzer (Foster City, CA, USA) [5].

Table 1. Primer sequences used for the polymerase chain reaction

\begin{tabular}{|c|c|c|c|}
\hline Nested PCR & Primer & Sequences $\left(5^{\prime}-3^{\prime}\right)$ & \\
\hline \multicolumn{4}{|c|}{ For full-length gag gene [6] } \\
\hline \multirow[t]{2}{*}{ First PCR } & $503 \mathrm{k}$ & 5'-CCKTCTGTTGTGTGACTCTGGTAA-3' & forward \\
\hline & 524 & 5'-CATTGTTTAACTTTTGGGCCATCC -3' & reverse \\
\hline \multirow[t]{3}{*}{ Second PCR } & $504 \mathrm{~F}$ & 5'-TCTCTAGCAGTGGCGCCCGAAC-3' & forward \\
\hline & 505 & 5'-GAGACATGGGTGCGAGAGCGT-3' & forward \\
\hline & 522 & 5'-ACTGTCCTACTTTGATAAAACCTC-3' & reverse \\
\hline \multicolumn{4}{|c|}{ For full-length pol gene $[2,7]$} \\
\hline \multirow[t]{4}{*}{ First PCR } & HXB2 & 5'-GTGGGAGAAATCTATAAAAGATGG-3' & forward \\
\hline & OBP2 & 5'-GAGACTCCCTGACCCAGATG-3' & reverse \\
\hline & OBP2k & 5'-GAGACTCCCTGACCCAGATG-3' & reverse \\
\hline & 550 & 5'-CCTAGTGGGATGTGTACTTCTGAA-3' & reverse \\
\hline \multirow[t]{5}{*}{ Second PCR } & PO1 & 5'-AAAATTGCAGGGCCCCTAGGA-3' & forward \\
\hline & PR3-1 & 5'-GAAGCAGGAGCCGATAGACA-3' & forward \\
\hline & OBP4 & 5'-CAATCATCACCTGCCATCTG-3' & reverse \\
\hline & $\mathrm{P} 2$ & 5'-AGGAAGGACACCAAATGAAAG-3' & forward \\
\hline & P16 & 5'-GGATKAGTGCTTTCATAGTGA-3' & reverse \\
\hline \multicolumn{4}{|c|}{ For vif, vpr, tat, rev, and vpu genes } \\
\hline \multirow[t]{2}{*}{ First PCR } & 545 & 5'-GCAGTACAAATGGCAGTATTCATC-3' & forward \\
\hline & $\mathrm{KMK} 2$ & 5'-ATGGGAATTGGTTCAAAGGA-3' & reverse \\
\hline \multirow[t]{2}{*}{ Second PCR } & $548 \mathrm{~F}$ & 5'-AGTGACATAAAAGTAGTRCCAAGAA-3' & forward \\
\hline & LA106 & 5'-TTCACTCTCATTGCCACT-3' & reverse \\
\hline \multicolumn{4}{|c|}{ For full-length env gene [5] } \\
\hline \multirow[t]{2}{*}{ First PCR } & OWE1 & 5'-TCATCAAGTTTCTCTATCAAAGCA-3' & forward \\
\hline & OWE2 & 5'-TCTGACTGGAAAGCCCACTT-3' & reverse \\
\hline Second PCR & OWE3 & 5'-GCAATATTAGCAATAGTTGTGTGG-3' & forward \\
\hline
\end{tabular}




\section{OWE4 5'-ATACTGCTCCCACCCCTTCT-3' reverse}

\begin{tabular}{cccc}
\hline \multicolumn{2}{c}{ For nef gene [4] } & & \\
\hline First PCR & Nef5'5 & 5'-AGGATTGTGGAACTTCTGGGAC-3' & forward \\
& LTR3 & 5'-AGGCTCAGATCTGGTCTAAC-3' & reverse \\
Second PCR & Nef3 & 5'-ATGGGTGGCAAGTGGTCAAA-3' & forward \\
& N10 & 5'-CGTCCAGAATTCGGAAAGTCCCCAGCGGAAAGT-3' & reverse \\
\hline
\end{tabular}

\subsection{Phylogenetic tree analysis}

In total, $70 \mathrm{FL}$ sequences were obtained from 20 patients with hemophilia. Sequences from 42 local controls and 1 subtype B infected patient were aligned against the HIV-1 subtype reference set from the HIV Sequence Database (http://hiv-web.lanl.gov/content/hiv-db/Subtype REF/align.html). Phylogenetic trees were constructed using the IQ tree with 1,000 bootstrap replicates [18].

\subsection{Viral signature pattern analysis (VESPA)}

The

VESPA

program

(http://www.hiv.lanl.gov/content/sequence/VESPA/vespa.html) was used to identify sites within each sequence group distinct from other groups [19].

\subsection{Statistical analysis}

Data are presented as means \pm standard deviation. Statistical significance was determined using Student's two-tailed t-tests, paired t-test, chi-square tests, Fisher's exact test, and Pearson's correlation coefficient using MedCalc. Results were deemed statistically significant when P-value was $<0.05$.

\subsection{Nucleotide sequence data}

The GenBank accession numbers for the sequences in this study are listed as follows: AF224507, AY839827, DQ054367, DQ295192-96, DQ837381，JQ316126-37, JQ341411, JQ429433， KF561435-43， KJ140245-66， KU869610, MK577478-81， MK871374, MG461319-22, MN237642-46, MN043576-607, MT101871, MT224125-27, MT559044-066, MT582420-24, MT679550-53, and MW405263-343. Subtype B sequences were randomly retrieved from the Los Alamos National Laboratory (LANL) HIV Database with the same number by year compared to KSB.

\section{Results}

\subsection{Origin of the KSB of subtype B}

A major contribution of this study is including the sequences of the earliest KSB-infected patients. We found that patient BGO diagnosed in 1988 was infected with KSB (MT559045). Hence, we obtained FLs in 36, 23, and 3 patients diagnosed in 1988-1991, 1992-1993, and after 1993, respectively (Table S1 and Figure 1). These 36 patients in 1988-1991 correspond to $84 \%$ of all 43 KSB-infected patients diagnosed in 1988-1991 [4]. 


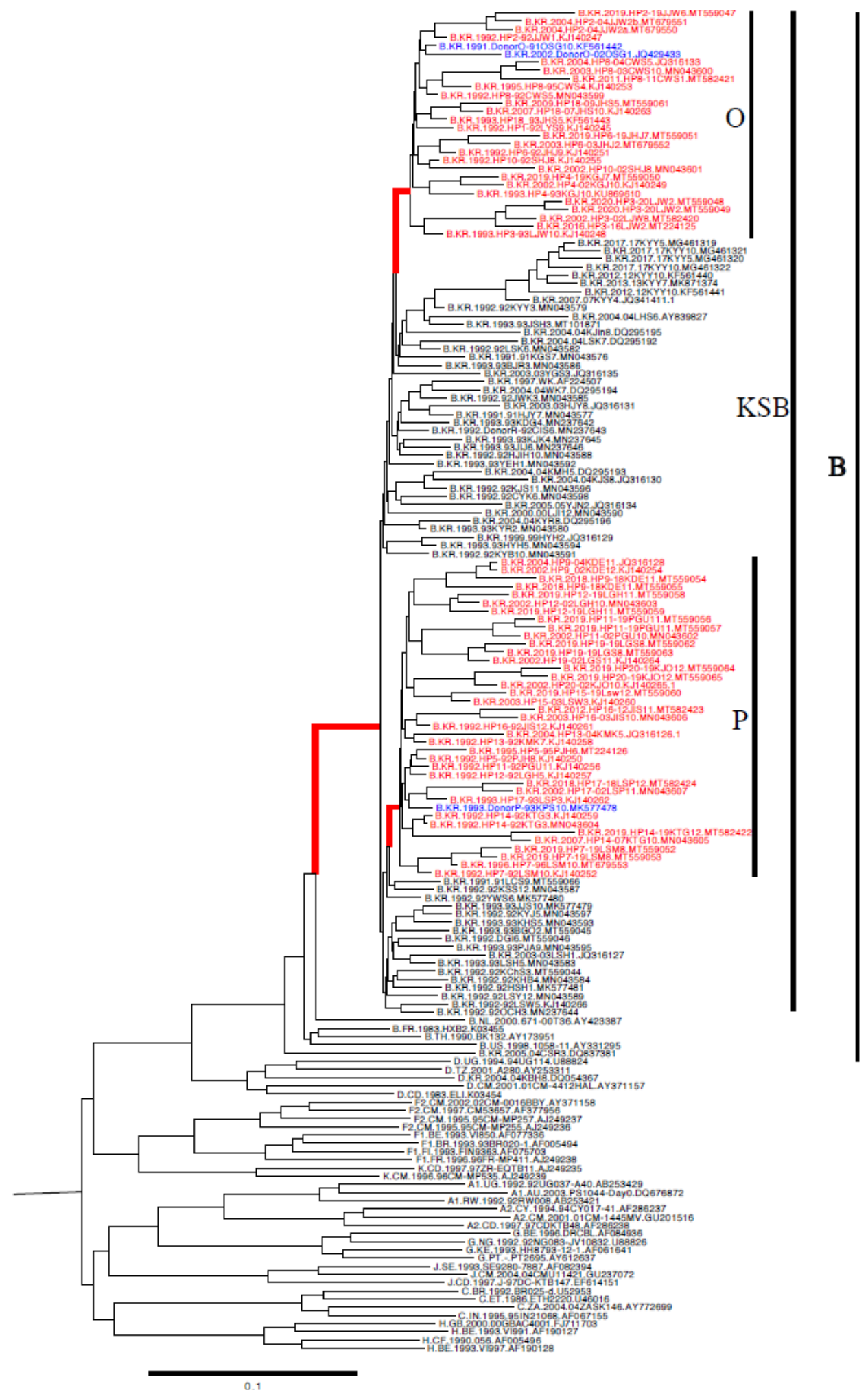


Figure 1. Phylogenetic tree analysis of the earliest near full-length sequences (about 8,615 bp from gag to nef gene) of 64 Korean patients infected with HIV-1 performed using the IQ-tree with 1,000 bootstrap replicates: twenty patients with hemophilia (HPs); 3 plasma donors (O, P, and R); 39 local controls infected with the Korean subclade of HIV-1 subtype B (KSB) and 2 non-KSB-infected patients. The upper 103 sequences belonged to KSB, and 2 sequences (05CSR3 and 04KBH8) belonged to subtypes B and D, respectively. In total, 9 and 13 patients, including donors $\mathrm{O}$ (Cluster $\mathrm{O}$ : donor $\mathrm{O}, 1-4,6,8,10$, and 18 as designated by red taxa) and $\mathrm{P}$ (Cluster $\mathrm{P}$ as designated by red taxa), strongly clustered within the KSB-infected local controls. The two digits before patient IDs and the one or two digits after patient IDs denote the year and month of sampling, respectively. The bootstrap values of the nodes for clusters $\mathrm{O}, \mathrm{P}$, and KSB (designated by bold in red) were $100 \%$ as determined by 1,000 bootstrap replicates. Furthermore, the sequences of each HP over 30 years also revealed $100 \%$ bootstrap values.

\subsection{Molecular epidemiologic data on the FL HIV-1 gene}

In $20 \mathrm{HPs}, 71 \mathrm{FLs}$ were obtained at 60 time points over $287 \pm 99$ months (about 24 years) during the outbreak in January 1990. We obtained 169 FLs from 65 patients. Of these, sequences were obtained from 2 or more samples collected on different dates from 30 patients, including 19 patients with hemophilia (Table S1). Phylogenetic analysis revealed that the earliest 62 FLs obtained from 62 patients (20 HPs and 42 local controls) belonged to KSB, whereas 2 FLs obtained from 2 patients belonged to subtypes B and D (Figure 1). The 62 KSB FLs were subdivided into several clusters, including two large clusters ("O," which comprised 9 sequences and " $\mathrm{P}$," which comprised 13 sequences) that included $20 \mathrm{HPs}$ and plasma donors $\mathrm{O}$ and $\mathrm{P}$. The bootstrap values of the nodes for clusters $\mathrm{O}, \mathrm{P}$, and KSB were all 100\% determined using 1,000 bootstrap replicates (Figure $1)$.

\subsection{Korean signature pattern amino acid residues}

We previously reported the signature pattern of amino acids at residues 12 and 26 in the Gag and Env proteins [5, 6]. Additionally, 8 and 9 signature pattern amino acids were determined in the Vif and Nef proteins, respectively [20, 21]. We found 31 novel Korean signature nucleotides in the pol gene compared with 31 sequences from 15 subtype B infected Korean patients (Table S2). Of those, 11 were nonsynonymous substitutions, and 20 were synonymous substitutions as compared to those in subtype B.

Overall, in the FLs sequences over 8,609-8,618-bp, the signature pattern analysis indicated 91 signature nucleotides (16, 21, 6, 2, 2, 2, 35, and 7 in gag, pol, vif, vpr, tat/rev, vpu, env, and nef genes, respectively; 1.05\%) that distinguish $20 \mathrm{HPs}$ and 42 local controls within KSB $(\mathrm{P}<0.05)$. In total, 48 and 43 signature nucleotides originated from clusters $\mathrm{O}$ and $\mathrm{P}$, respectively. Of those, only six positions in gag, pol, vif, and env genes contained $100 \%$ specific nucleotide(s) positions in clusters $\mathrm{O}$ and $\mathrm{P}$ [2-6] as compared to $0 \%$ in local controls (Table S3).

\subsection{Sequence identities of HPs compared to plasma donors $O$ and $P$}

The earliest FLs from two donors $\mathrm{O}$ and $\mathrm{P}$ were 8,606 bp and 8,618 bp, respectively. The sequence identity between the earliest sequences determined in October 1991 and the last sequences determined in January $2002(8,627 \mathrm{bp})$ from donor $\mathrm{O}$ was $96.5 \%$. The sequence similarity in cluster $\mathrm{O}$ between the earliest sequences from donor $\mathrm{O}$ and the sequence of each HP averaged $97.7 \% \pm 0.8 \%$. In cluster $\mathrm{P}$, the sequence similarity between the earliest sequences from donor $\mathrm{P}$ and each $\mathrm{HP}$ was $97.4 \% \pm 1.4 \%$.

We determined the correlation between the sampling intervals after the outbreak (January 1990) and the number of nucleotide differences observed, relative to those of the corresponding plasma donor. Four patients who were first sampled in 2002 exhibited the lowest sequence identity (Figure 2A). The sequence identity dropped significantly over time from the outbreak. The lowest sequence identity was $89.8 \%$ at 153 months in October 2002 in HP-20. In 19 HPs, sequencing was repeatedly performed with approxi- 
mately a 128-month interval. The overall correlation coefficient, $\gamma$, was estimated to be $-0.85(\mathrm{P}<0.001)$ (Figure 2A).

When we analyzed $40 \mathrm{FLs}$ before the administration of cART, the $\gamma$ was $0.77(\mathrm{P}<$ 0.001) (Figure 2B), whereas $\gamma$ was -0.01 at the administration of cART compared to the sequences just before the administration of cART (Figure 2C). In the same context, the correlations were also significant between the sampling year and sequence length before the administration of cART in $20 \mathrm{HPs}(r=0.79 ; \mathrm{P}<0.001)$ (Figure 2D).

In addition, we found this phenomenon in 42 local controls patients without hemophilia $(r=0.38$; $\mathrm{P}<0.01)$ (Figure $2 \mathrm{E})$, and the correlation was higher in 12 local controls with sequences determined at 2 time points before cART $(r=0.61 ; \mathrm{P}=0.001)$.
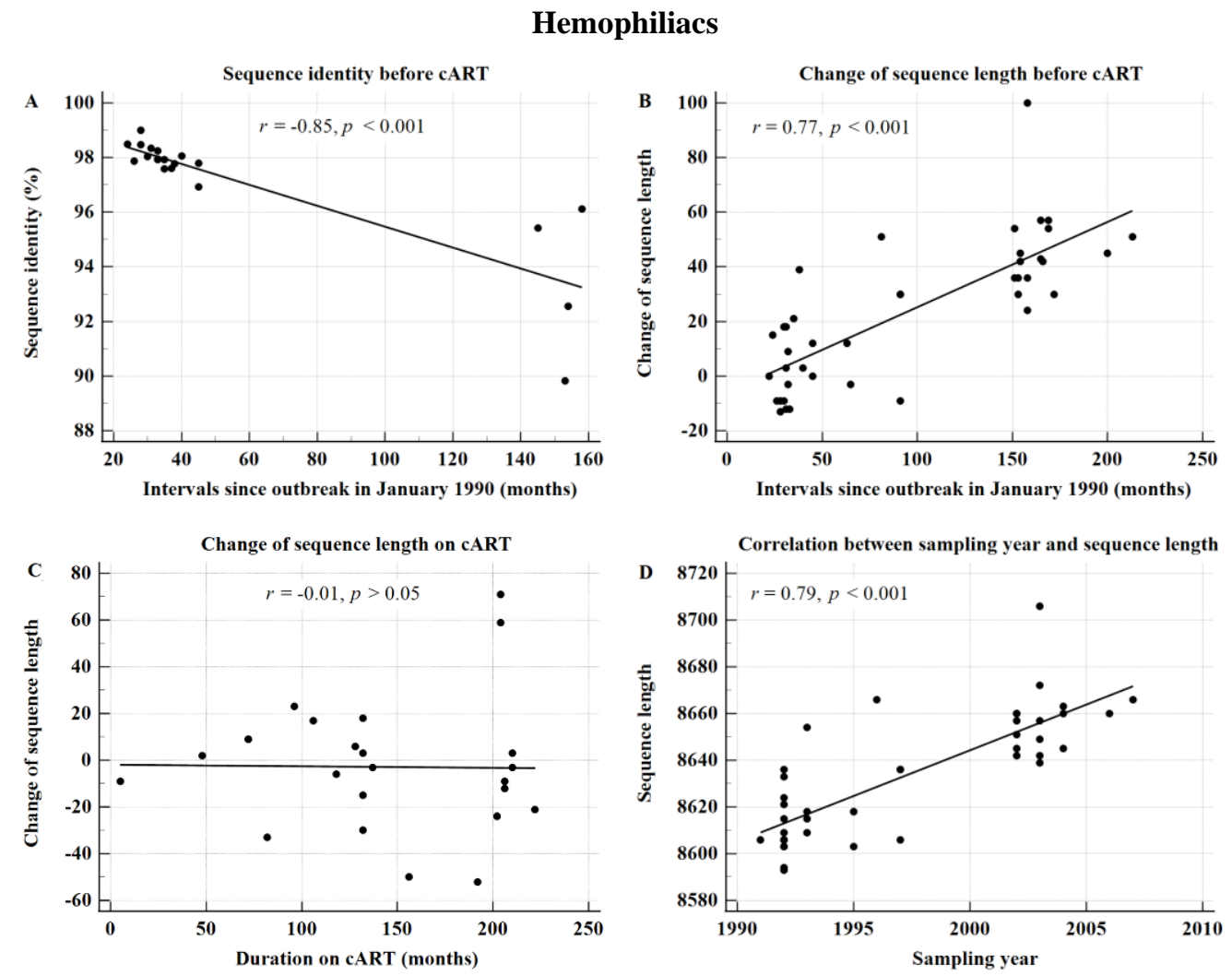

Local controls
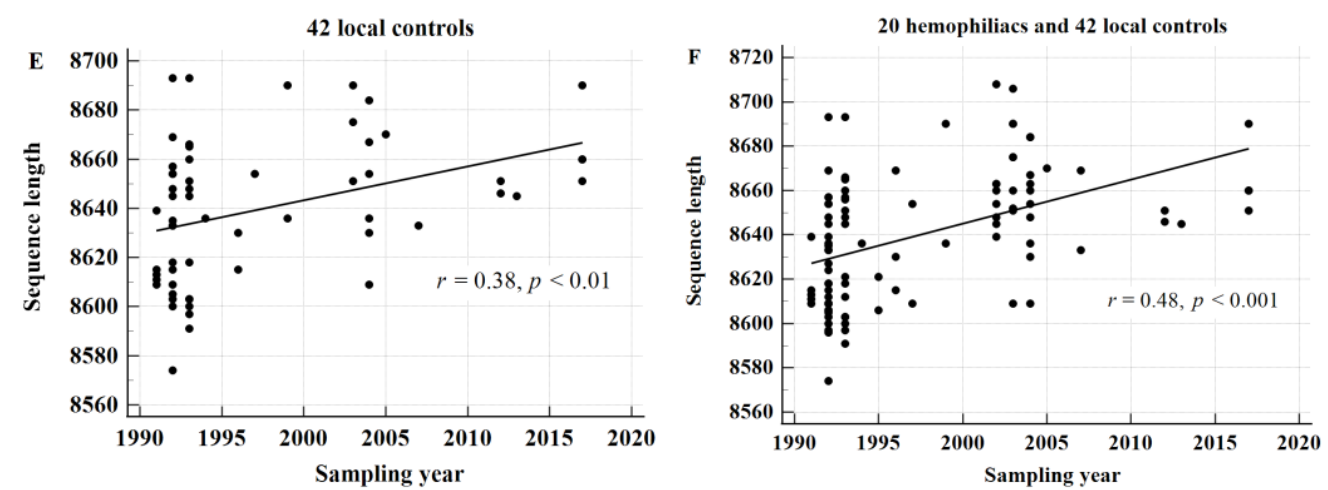

Figure 2. Sequence length increases over time and sequence identity decreases in KSB-infected patients. (A - C): Sequence identity and full-length (FL) sequences according to the interval since the outbreak in January 1990 in 20 HPs. (A) Correlation between the intervals from the outbreak to sampling and the sequence identity of each earliest FL sequence before cART in the 20 patients with hemophilia, compared with the corresponding plasma donor's earliest sequence (B) The correlation coefficient (CC), $\gamma$, was 0.77 for 
the 39 FLs before cART $(P<0.001)$. (C) CC, $\gamma$ was -0.01 on cART. (D - F), Significant increase in sequence length by sampling year in patients with hemophilia (D), $42 \mathrm{KSB}$-infected local controls $(n=60)(E)$, and all $62 \mathrm{KSB}$-infected patients $(\mathrm{F})$.

\subsection{Sequence length of HIV-1 KSB significantly increases over time before cART}

Among the earliest $20 \mathrm{FLs}$ in $20 \mathrm{HPs}$, we excluded $4 \mathrm{HPs}$ because the sequences were obtained after ten years since the outbreak in January 1990. The earliest sequences obtained over $32 \pm 5$ months from the outbreak revealed 8,614 \pm 17 nucleotides $(n=16)$ with an increase of $4.1 \pm 16$ nucleotides per year than the corresponding donor's sequences. The second sequences obtained more than 5 years after the outbreak were determined from samples collected from 17 HPs before cART $(n=23)$. This revealed $8,650 \pm$ 22 nucleotides with a significant increase of $39 \pm 22$ nucleotides over $143 \pm 41$ months from the outbreak $(\mathrm{P}<0.0001)$; however, first sequences of donors $\mathrm{O}$ and $\mathrm{P}$ obtained in 1991 and 1993 were used.

We previously found that sequence length significantly increased over time before cART (Figure 2B). The sequence length was analyzed by genes to confirm which gene demonstrated increased sequence length. The increase could be attributed to a significant increase in env $(\mathrm{P}<0.0001)$ and nef $(\mathrm{P}<0.01)$ genes (Table 2$)$. The increase originated in the variable regions of both genes.

Table 2. Changes of the sequence length of HIV-1 over $110 \pm 43$ months by genes in patients with hemophilia

\begin{tabular}{|c|c|c|c|c|c|c|c|c|c|c|}
\hline $\begin{array}{c}\text { Sequences } \\
\text { with in- } \\
\text { tervals } \\
\end{array}$ & gag & pol & vif & vpr & tat & rev & vpu & env & nef & FL \\
\hline $\begin{array}{l}\text { First se- } \\
\text { quences } \\
(\mathrm{n}=16)\end{array}$ & $1503 \pm 0$ & $3012 \pm 0$ & 579 & 291 & 215 & 76 & $247 \pm 2$ & $2560 \pm 19$ & $623 \pm 2$ & $8617 \pm 20$ \\
\hline $\begin{array}{c}\text { Second } \\
\text { sequences } \\
(\mathrm{n}=16)\end{array}$ & $1504 \pm 3$ & $3011 \pm 3$ & 579 & 291 & 215 & 76 & $247 \pm 2$ & $2592 \pm 22$ & $627 \pm 8$ & $8657 \pm 23$ \\
\hline P-value & NS & NS & NS & NS & NS & NS & NS & $<0.001$ & $<0.05$ & $<0.0001$ \\
\hline
\end{tabular}

There was an interval of about $110 \pm 43$ months between the sequences obtained at the two time points. NS; not significant. P-value by paired T-test.

In contrast, we analyzed the change of sequence length within each HP. The sequence length increased by $40 \pm 30$ nucleotides over $110 \pm 43$ months between the first and last sequences in $16 \mathrm{HPs}$, with sequences determined at $\geq 2$ time points before cART $(\mathrm{P}<0.0001)$ (Table 2). This corresponds to an annual increase of 4.4 nucleotides and a requirement of 47 and 32 years to reach the mean length of SIVsm and HIV-2, respectively.

The sequence length in the $e n v$ gene increased by $30 \pm 29$ nucleotides during the same period $(\mathrm{P}<0.001)$.

In contrast, the sequence length decreased by $7.6 \pm 25$ nucleotides over $178 \pm 48$ months at the administration of cART in 17 HPs compared to the sequence length determined just before the administration of cART (Figure S1).

To confirm whether this phenomenon occurs in patients without hemophilia, we analyzed sequences in $12 \mathrm{KSB}$ infected patients with the sequences determined at $\geq 2$ time points among 42 local controls. There was also a significant increase in sequence length between the 2 FLs over $104 \pm 43$ months ( $33 \pm 29$ nucleotides) $(\mathrm{P}<0.01)$. Of the increase of $33 \pm 29$ nucleotides, the sequence length in the env gene increased by $24 \pm 25$ 
nucleotides over $104 \pm 43$ months $(\mathrm{P}<0.05)$, whereas the increase in the nef gene was not significant.

Combining $20 \mathrm{HPs}$ with 42 local controls, significant correlations between 100 FLs and the sampling year $(\mathrm{P}<0.001)$ (Figure $2 \mathrm{~F})$, and sequence length of the env and the sampling year $(r=0.45, \mathrm{P}<0.001)$ were also observed, whereas no significant correlation was observed with cART.

Additionally, we found a significant correlation even in individual patients with long-term slow progression (Figure 3).
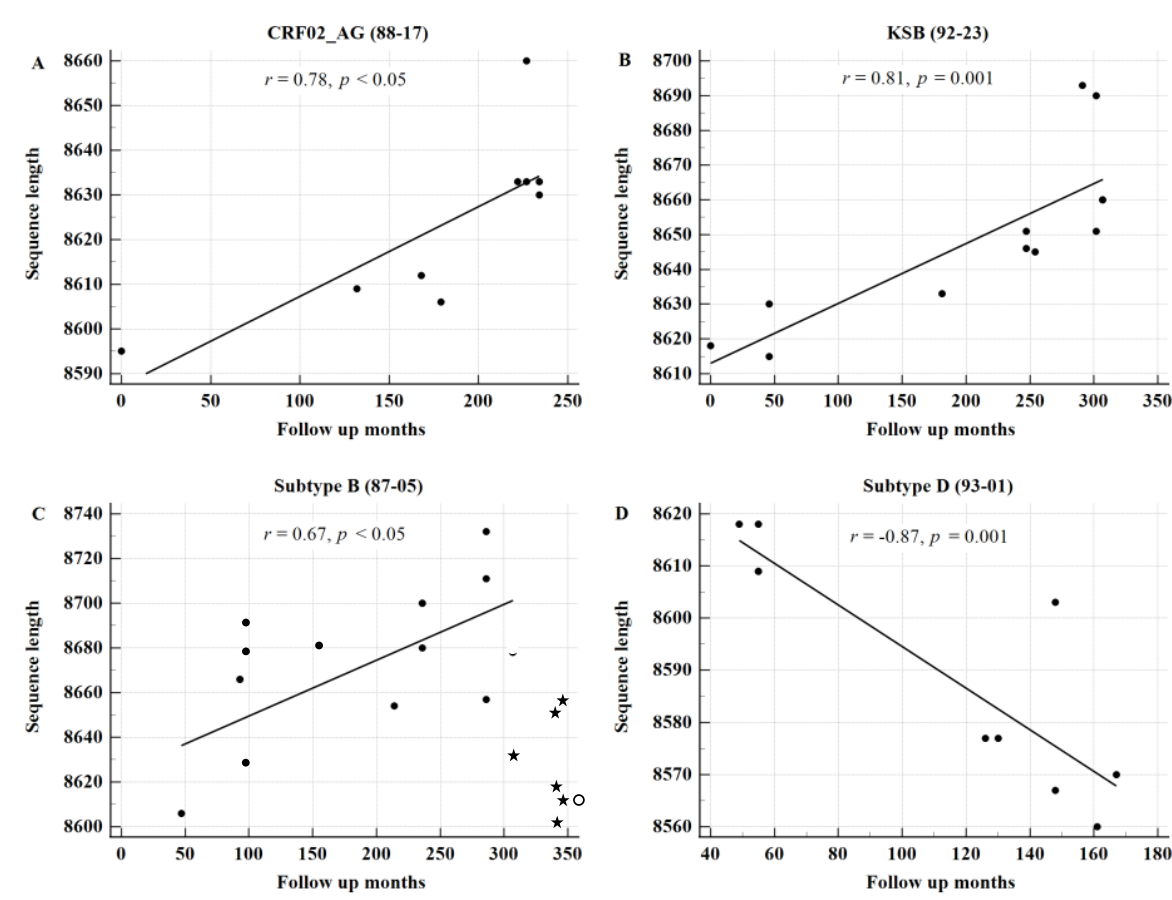

Figure 3. FLs increased over time in 3 long-term non progressors (LTNP), whereas it decreased in subtype D infected patient. The correlation coefficient was significant between sequence length and duration of infection in 3 LTNPs over 25 years (up to 294,307 and 286 months). Patients infected with CRF02_AG (A), KSB (B), and subtype B (C) were diagnosed with HIV-1 infection in 1988, 1992, and 1987, respectively. Interestingly, the sequence length in patient infected with subtype B significantly decreased from $8,697 \pm 26(n=6)$ just before 286 months (March 2011) to 8,623 $\pm 24(n=6$; marked as black star) after 286 months when plasma RNA copy significantly increased $(\mathrm{P}<0.001)$ [37]. The sequence length significantly decreased after 307 months. In contrast, it was inversely significant in the patient 93-01 who was diagnosed in December 1992 (D). The patient's first sexual contact and diagnosis of pulmonary tuberculosis were observed in 1988 and 1989, respectively. He was treated with Korean Red Ginseng (12,720 g) from April 1993 to August 2004 [33]. Thus, despite the most rapidly progressing subtype D infection, he remained healthy at least for 12 years [33]. The correlation analysis did not include the data marked as star and white circle on cART. The sequence length at the administration of cART decreased in patients 88-17 and 87-05.

\subsection{Sequence length in HIV-1 subtype B also significantly increased over time}

To confirm whether the sequence length increases in subtypes other than KSB, we randomly selected 64 FLs subtype B from the LANL HIV Database from 1983 to 1998. The correlation between sampling year and sequence length was significant for FLs ( $\mathrm{r}=$ $0.43, \mathrm{P}<0.001)$ (Figure 4$)$, env $(\mathrm{r}=0.26, \mathrm{P}<0.05)$, and nef $(\mathrm{r}=0.30, \mathrm{P}<0.05)$ genes, respectively. 


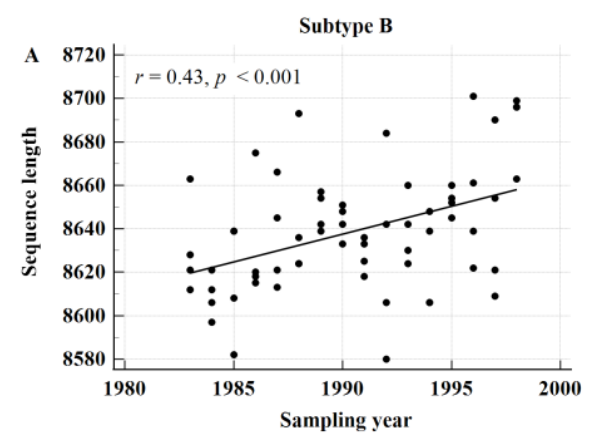

Figure 4. Sequence length also increases over time in subtype B infected patients. A total of 64 FLs of Western subtype B were randomly selected from Los Alamos National Laboratory Database over 16 years from 1983 to 1998 (evenly 4 per year).

\subsection{Clinical significance of the increase in sequence length in HIV-1 subtype B}

Several reports have been published stating that V1-V2 envelope loops and env sequences length increase by $\sim 1 \%$ per year in the early phases of typical infections [22-24]. There had been several reports on the elongation of the V2 region in long-term nonprogressors (LTNPs) [25-28]. We analyzed the correlation between the number of amino acids in the V2 region and the duration since the diagnosis of KSB and subtype B infection. Consistent with the increase in sequence length over time, there were significant correlations between the two aforementioned factors $(n=213, r=0.60, P<0.001)$ as well as between CD4+ $\mathrm{T}$ cell count and the number of amino acids in the V2 region in 75 patients $(n=213, r=-0.17, P<0.05)$. Additionally, there were significant inverse correlations between FLs and CD4+ T cell count $>100 / \mu \mathrm{L}$ in all 65 patients $(\mathrm{r}=-0.30, \mathrm{P}=0.001)$ and between the sequence length of the env gene and CD4+ T cell counts $>100 / \mu \mathrm{L}$ before cART $(\mathrm{r}=-0.34, \mathrm{P}<0.001)($ Table S1).

\section{Discussion}

This study provides evidence suggesting that sequence length increases over time as demonstrated by extensive sequence data collected from 20 patients with hemophilia with a well-known history and KSB-infected local controls. Due to well-documented primary HIV-1 infection [1-7] and extensive sequence data obtained over 30 years from 20 HPs with a common source of HIV-1, we were able to confirm that FL significantly increases over time, and the strength of correlation was stronger in $20 \mathrm{HPs}$ (Figure 2D) than that in local controls (Figure 2E). Otherwise stated, the more homogeneous the cohort, the higher strength of correlation.

To our knowledge, this is the first report on the association between sequence length in FLs level and duration of infection. At any given time, viral populations will be dominated by those strains that are most fit at that time [25]. However, several reports have focused on V1 and V2 elongation in an elite controller [26-29], and consistent usage of the CCR5 coreceptor [30]. As a virulence gene, the sequence length of the nef gene compared to other genes is significantly shortened in HIV-1 and SIVcpz than that in HIV-2 and SIVsm [31] (Figure S3), implying pathogenicity [32].

The decreasing rate of CD4+ T cell counts is faster in subtype $\mathrm{D}$ than that in subtype B $[15,33]$. FL in subtype D was significantly shorter than those in subtypes B, KSB, C, and G (Figure S2). This might be associated with the shorter survival time required for subtype $\mathrm{D}$ viruses to adapt in vivo than that required for other subtypes. Thus, there was no correlation between the sampling year and sequence length in subtype D (data 
not shown). When we compared the sequence length in the env gene among three subtypes, sequence length in subtype $B(n=64)$ was significantly longer $(2,575 \pm 21)$ than $2,547 \pm 20$ in subtype D (patient 93-01) $(\mathrm{P}<0.0001)$. FLs in subtypes B $(8,639 \pm 28$ ) were significantly longer than those in subtype $\mathrm{D}(\mathrm{n}=10,8,589 \pm 19, \mathrm{P}<0.0001)$.

It is known that sequence length is significantly longer in SIVsm than in HIV-2. The increase results from the increase in env and nef genes. Taken together, these data suggest that the longer sequence length (SIVsm $>$ HIV-2 $>$ SIVcpz $>$ HIV-1 subtypes B/KSB $>$ D) (Figure S2) corresponds to a longer survival. When we translated the longevity of chimpanzees and sooty mangabeys into human beings (60 years), there was a significant correlation between the sequence length and survival duration $(r=0.90 \sim r=0.88, \mathrm{P}<$ 0.05).

It is important to reemphasize that viruses adopt a symbiotic pathway rather than killing the host or evolving toward attenuated pathogenicity [25]. In fact, the replicative capacity of HIV-1 in the 2000s was significantly lower than that of the virus in the 1980s [34]. Consequently, the survival period was longer in the infected people in the 2000s; however, various factors might be involved. The replicative fitness by subtype is determined by the following order: $\mathrm{D}>\mathrm{A}>\mathrm{C}$ [35]. In this respect, it is possible to understand the increase in sequence length as a strategy or evolutionary direction for the virus to adapt under the pressure exerted by the immune system of the host and coexist with the host.

The increase in the sequence length implies that the longer the coding sequence (CDS) length, the lower the density of ribosomes, resulting in less efficient protein synthesis [36] and fewer virus copies. This may be the reason why an increase in sequence length, such as V1 or V2 elongation, occurs in the elite controller or LTNP [25-27] as observed in this study. However, the prognosis of patients with SIVsm and HIV-2 exhibiting longer CDS is better than that of HIV-1. Probably, the increase in CDS in HIV-1 over time might be related to attenuated pathogenicity and a different evolutionary direction.

This study had a few limitations. First, sera sampled from different individuals at various time points were used, and the time lag between primary infection and sampling was particularly long over 10 years in four HPs. Second, sequences before 2000 and after 2000 were obtained from serum and PBMCs, respectively.

In this study, the sequence length of HIV-1 increased by 4.4 nucleotides per year before cART. When viruses were transmitted to another patient, among quasispecies, the possibility of infection by HIV-1 with a shorter sequence might be higher than that by HIV-1 with long sequences. Thus, at the population level, the accumulation effect of the increase in sequence length might be slower than that at the individual level because of the bottleneck effect. Our novel data suggest that the increase in CDS over time might be an evolutionary direction.

Supplementary Materials: The following are available online at www.mdpi.com/xxx/s1, Figure S1. Comparison of the increase in the length of the full-length HIV-1 sequence before cART and upon cART in 16 patients with hemophilia; Figure S2. The order of length in the near full-length sequence of HIV-1 (from subtype D to G), SIVcpz, HIV-2, and SIVsm; Figure S3. Comparison of the length of the Nef proteins of HIV-1, SIVcpz, HIV-2, and SIVsm.

Author Contributions: Y.-K.C. designed the experiments. Y.-K.C. and J.-E.K. performed the experiments.Y.-K.C., and B.T.F analyzed the data and wrote the paper.

Funding: This work was supported by a grant from the Korean Society of Ginseng (2012-2020).

Institutional Review Board Statement: The institutional review board of the Asan Medical Center approved this study's conduct (Code 2012-0390, 4 June 2012). All subjects gave their informed consent for inclusion before they participated in the study. The study was conducted in accordance with the Declaration of Helsinki.

Informed Consent Statement: Any research article describing a study involving humans should contain this statement. Please add "Informed consent was obtained from all subjects involved in the study." OR "Patient consent was waived due to REASON (please provide a detailed justifica- 
tion)." OR “Not applicable." for studies not involving humans. You might also choose to exclude this statement if the study did not involve humans.

Written informed consent for publication must be obtained from participating patients who can be identified (including by the patients themselves). Please state "Written informed consent has been obtained from the patient(s) to publish this paper" if applicable.

Data Availability Statement: In this section, please provide details regarding where data supporting reported results can be found, including links to publicly archived datasets analyzed or generated during the study. Please refer to suggested Data Availability Statements in section "MDPI Research Data Policies" at https://www.mdpi.com/ethics. You might choose to exclude this statement if the study did not report any data.

Acknowledgments: We thank the patients with hemophilia for their cooperation. The authors would like to thank editage (www.editage.co.kr) for the English language review.

Conflicts of Interest: The authors declare no conflict of interest.

\section{References}

1. Cho, Y.K.; Foley, B.T.; Sung, H; Kim, Y.B.; Kim, J.H. Molecular epidemiologic study of a human immunodeficiency virus 1 outbreak in hemophiliacs B infected through clotting factor 9 after 1990. Vox Sang. 2007, 92, 113-120.

2. Cho, Y.K.; Jung, Y.S.; Foley, B.T.; Phylogenetic analysis of full-length pol gene from Korean hemophiliacs and plasma donors infected with Korean subclade B of HIV-1. AIDS Res. Hum. Retrovir. 2011, 27, 613-621.

3. Cho, Y.K.; Jung, Y.; Lee, J.S.; Foley, B.T. Molecular evidence of HIV-1 transmission in 20 Korean individuals with hemophilia; phylogenetic analysis of vif gene. Haemophilia 2012, 18, 291-299.

4. Cho, Y.K.; Kim, J.E.; Foley, B.T. Phylogenetic analysis of the earliest nef gene from hemophiliacs and local controls in Korea. BioRes. Open Access 2012, 1, 41-49.

5. Cho, Y.K.; Kim, J.E.; Jeong, D.; Foley, B.T. Signature pattern analysis for the full-length env gene of the earliest Korean subclade B of HIV-1: outbreak among Korean hemophiliacs. Virus Genes 2017, 53, 789-796.

6. Cho, Y.K.; Kim, J.E.; Foley, B.T. Genetic analysis of the full-length gag gene from the earliest Korean subclade B of HIV-1: an outbreak among Korean hemophiliacs. Viruses 2019, 11:545.

7. Cho, Y.K.; Kim, J.E.; WOO, J.H.; Korean Red Ginseng increases defective pol gene in peripheral blood mononuclear cells of HIV-1-infected patients; inhibition of its detection during ginseng-based combination therapy. J Ginseng Res. 2019, 43, 684-691.

8. Leitner, T.; Escanilla, D.; Franzén, C.; Uhlén, M.; Albert, J. Accurate reconstruction of a known HIV-1 transmission history by phylogenetic tree analysis. Proc. Natl. Acad. Sci. U S A 1996, 93, 10864-10869.

9. Novitsky, V.; Moyo, S.; Lei, Q.; DeGruttola, V.; Essex, M. Importance of viral sequence length and number of variable and informative sites in analysis of HIV clustering. AIDS Res. Hum. Retrovir. 2015, 31, 531-542.

10. Daniels, R.S.; Kang, C.; Patel, D.; Xiang, Z.; Douglas, N.W.; Zheng, N.N.; Cho, H.W.; Lee, J.S. An HIV type 1 Subtype B founder effect in Korea: gp160 signature patterns infer circulation of CTL-escape strains at the population level. AIDS Res. Hum. Retrovir. 2003, 19, 631-641.

11. Korber, B.; Myers, G.; Signature pattern analysis: a method for assessing viral sequence relatedness. AIDS Res. Hum. Retrovir. 1992, 8, 1549-1560.

12. Korber, B.T.; Foley, B.; Gaschen, B.; Kuiken, C. Epidemiological and immunological implications of the global variability of HIV-1. In: Pataleo, G.; Walker, B.D. (eds), Humana Press, Totowa, NJ. 2001; pp. 1-32, ISBN 978-1-61737-128-8.

13. Junqueira, D.M.; Almeida, S.E. HIV-1 subtype B: traces of a pandemic. Virology 2016, 495, 173-184.

14. Kim, M.S.; Jang, S.Y.; Park, C.S.; Lee, K.M.; Lee, D.H.; Lee, C.H. Timing and evolution of the most recent common ancestor of the Korean clade HIV subtype B based on nef and vif sequences. J. Microbiol. 2009, 47, 85-90.

15. Easterbrook, P.J.; Smith, M.; Mullen, J.; O'Shea, S.; Chrystie, I.; de Ruiter, A.; Tatt, L.D.; Geretti, A.M.; Zuckerman, M. Impact of HIV-1 viral subtype on disease progression and response to antiretroviral therapy. J. Int. AIDS Soc. 2010, 3, 13:4, doi: 10.1186/1758-2652-13-4.

16. Cho, Y.K.; Kim, J.E.; Foley, B.T. Phylogenetic analysis of near full-length HIV-1 genomic sequences from 21 Korean individuals. AIDS Res. Hum. Retrovir. 2013, 29, 738-743.

17. Cho, Y.K.; Sung, H.; Bae, I.G.; Oh, H.B.; Kim, N.J.;Woo, J.H.; Kim, Y.B. Full sequence of HIV type 1 Korean subtype B in an AIDS case with atypical seroconversion: TAAAA at TATA box. AIDS Res. Hum. Retrovir. 2005, 21, 961-964.

18. Nguyen, L.T.; Schmidt, H.A.; Haeseler, A.; Minh, B.Q. IQ-TREE: A fast and effective stochastic algorithm for estimating maximum-likelihood phylogenies. Mol. Bio. Evol. 2015, 32, 268-274.

19. Ou, C.Y.; Ciesielski, C.A.; Myers, G.; Bandea, C.I.; Luo, C.C.; Korber, B.T.; Mullins, J.I.; Schochetman, G.; Berkelman, R.L.; Economou, A.N.; et al. Molecular epidemiology of HIV transmission in a dental practice. Science 1992, 256, $1165-1171$.

20. Park, C.S.; Kim, M.S.; Lee, S.D.; Kim, S.S.; Lee, K.M.; Lee, C.H. Molecular phylogenetic analysis of HIV-1 vif gene from Korean isolates. J. Microbiol. 2006. 44, 655-659. 
21. Park, C.S.; Lee, D.H.; Lee, K.M.; Lee, C.H. Characterization and signature pattern analysis of Korean clade HIV-1 using nef gene sequences. J. Microbiol. 2008, 46, 88-94.

22. Shankarappa, R.; Margolick, J.B.; Gange, S.J.; Rodrigo, A.G.; Upchurch, D, Farzadegan H, Gupta, P.; Rinaldo, C.R.; Learn, G.H.; et al. Consistent viral evolutionary changes associated with the progression of human immunodeficiency virus type 1 infection. J. Virol. 1999, 73, 10489-10502.

23. Sagar, M.; Wu, X.; Lee, S.; Overbaugh, J. Human immunodeficiency virus type 1 V1-V2 envelope loop sequences expand and add glycosylation sites over the course of infection, and these modifications affect antibody neutralization sensitivity. J. Virol. 2006, 80, 9586-9598.

24. Bunnik, E.M.; Euler, Z.; Welkers, M.R.; Boeser-Nunnink, B.D.; Grijsen, M.L.; Prins, J.M.; Schuitemaker, H. Adaptation of HIV-1 envelope gp120 to humoral immunity at a population level. Nat. Med. 2010, 16, 995-997.

25. Malim, M.H.; Emerman, M.; HIV-1 sequence variation, drift, shift, and attenuation. Cell 2001, 104, 469-472.

26. Silver, Z.A.; Dickinson, G.M.; Seaman, M.S.; Desrosiers, R.C. A highly unusual V1 region of Env in an elite controller of HIV infection. J. Virol. 2019, 93, e00094-19, doi: 10.1128/JVI.00094-19.

27. Shioda, T.; Oka, S.; Xin, X.; Liu, H.; Harukuni, R.; Kurotani, A.; Fukushima, M.; Shiino, T.; Takebe, Y.; Lwamoto, A.; et al. In vivo sequence variability of human immunodeficiency virus type 1 envelope gp120: association of V2 extension with slow disease progression. J. Virol. 1997, 71, 4871-4881.

28. Wang, B.; Spira, T.J.; Owen, S.; Lal, R.B.; Saksena, N.K. HIV-1 strains from a cohort of American subjects reveal the presence of a V2 region extension unique to slow progressors and non-progressors. AIDS 2000, 14, 213-223.

29. Masciotra, S.; Owen, S.M.; Rudolph, D.; Yang, C.; Wang, B.; Saksena, N.; Spira, T.; Dhawan, S.; Lal, R.B. Temporal relationship between V1V2 variation, macrophage replication, and coreceptor adaptation during HIV-1 disease progression. AIDS 2000, 16, 1887-1898.

30. Daniels, R.S.; Wilson, P.; Patel, D.; Longhurst, H.; Pattersom, S. Analysis of full-length HIV type 1 env genes indicates differences between the virus infecting $\mathrm{T}$ cells and dendritic cells in peripheral blood of infected patients. AIDS Res. Hum. Retrovir. 2004, 20, 409-413.

31. Hirao, K.; Andrews, S.; Kuroki, K.; Kusaka, H.; Tadokoro, T.; Kita, S.; Ose, T.; Rowland-Jones, S.L.; Maenaka, K. Structure of HIV-2 Nef reveals features distinct from HIV-1 involved in immune regulation. iScience 2020, 24, doi:10.1016/j.isci.2019.100758.

32. Schindler, M.; Münch, J.; Kutsch, O.; Li, H.; Santiago, M.L.; Bibollet-Ruche, F.; Müller-Trutwin, M.C.; Novembre, F.J.; Peeters, M.; Courgnaud, V.; et al. Nef-mediated suppression of T cell activation was lost in a lentiviral lineage that gave rise to HIV-1. Cell 2006, 125, 1055-1067.

33. Cho, Y.K.; Kim, J.E.; Lee, S.U.; Foley, B.T.; Choi, B.S. Impact of HIV-1 subtype and Korean Red Ginseng on AIDS progression: comparison of subtype B and subtype D. J. Ginseng Res 2019, 43, 312-319.

34. Ariën, K.K.; Troyer, R.M.; Gali, Y.; Colebunders, R.L.; Arts, E.J.; Vanham, G. Replicative fitness of historical and recent HIV-1 isolates suggests HIV-1 attenuation over time. AIDS 2005, 19, 1555-1564.

35. Venner, C.M.; Nankya, I.; Kyeyune, F.; Demers, K.; Kwok, C.; Chen, P.L.; Rwambuya, S.; Munjoma, M.; Chipato, T.; Byamugisha, J.; et al. Infecting HIV-1 subtype predicts disease progression in women of sub-Saharan Africa. EBioMedicine 2016, 13, 305-314.

36. Fernandes, L.D.; de Moura, A.P.S.; Ciandrini, L. Gene length as a regulator for ribosome recruitment and protein synthesis: theoretical insights. Sci. Rep. 2017, 7, 17409, doi: 10.1038/s41598-017-17618-1.

37. Cho, Y.K.; Kim, J.E.; Woo, J.H. Genetic defects in the nef gene are associated with Korean Red Ginseng intake: monitoring of nef sequence polymorphisms over 20 years. J Ginseng Res. 2017, 41, 144-150. 